himself points out, accompanied by more than an added complexity in the movements of this particular district. There is a ne'w "figure" to equilibrate. It might also be suggested that there is something new in the location of eyes and semicircular canals at the end of so long a flail.

No one interested in the central nervous system can read Prof. Bolk's book without attention or without criticism.

J. S. Macdonald.

\section{PARTIAL DIFFERENTIAL EQUATIONS.}

Theory of Differential Equations. By Dr. A. R. Forsyth, F.R.S. Vol. v., pp. $x x+478$; vol. vi., pp. xiv +596 . (Cambridge: University Press, I906.) Price $25 s$, net.

THE appearance of these volumes marks the happy conclusion of a work undertaken, as the author reminds us in his preface, twenty-one years ago. Doubtless it would have been finished earlier had it not been for unavoidable interruptions; but the delay must have brought its compensations, because many most interesting developments are of recent date.

Vol. v. deals with equations of the first order, and immediately suggests two reflections-one that Lie has made the most important contribution to the subject since the publication of Jacobi's memoirs, and the other that it is a great help to have such an outline of Lie's theory, with Mayer's simplifications, as that given in chapter ix. The Jacobian theory, too, with Mayer's developments, is given in chapters iii., iv. in a very attractive and readable form. Chapters vi., vii., viii. are mainly concerned with characteristics, and embody much of the work of Cauchy, Monge, Lie, and Darboux, as well as original contributions by Prof. Forsyth himself.

It may be a rather far-fetched comparison, but there does appear to be a kind of analogy between the achievements of von Staudt and Lie. Von Staudt's treatise on projective geometry does not contain a single diagram, but it is beyond question the most masterly work on the subject. Lie is almost, if not quite, as chary of graphical illustration, but the spirit of his work is geometrical throughout, and he stands in the same sort of relation to Monge that von Staudt does to Steiner. It is most interesting to see how the canonical equations of dynamics (pp. 398-406) are illuminated by the theory of contact transformations; and, again, it is mainly. Lie's ideas which have prepared the way for a thorough discussion of all the solutions of a partial differential equation, including the special integrals which do not come into the ordinary classification.

The great advance which has been made arises from considering a differential equation, not merely as representing a property of a function assumed to exist, but as defining an aggregate of elements which are most vividly realisable in a geometrical form. In partial differential equations of the first order these elements may be taken to be tiny fragments of planes scattered about in space; the differential equation de- fines the system of elements, and a complete integral, if it exist, represents the collecting of the elements into surfaces which form a family. In Clebsch's treatise on geometry, there is a chapter on connexes to which he evidently attached importance, and which has obvious relations, not only to mixed concomitants, but also to ordinary differential equations. If it has not been already done, it might be worth while to see whether something might not be made out of these relations; Clebsch's work has, of late, rather suffered neglect. Again, it may be suggested that in dealing with partial differential equations of the second order it might be helpful to associate with given values $(x, y, z, p, q, r, s, t)$ a fragment of a surface of the second order, just as a fragment of a plane is associated with $(x, y, z, p, q)$. That fragments of this kind are less likely to be associable so as to form surfaces than corresponding plane elements is tolerably plain, and partly accounts for the increasing difficulty of treating equations of the second order without making particular assumptions.

Vol. vi. of the present work is practically devoted to partial differential equations of the second order. Thus we have chapters on Laplace's linear equation, with the elegant developments of Darboux, Moutard, and others; the methods of Monge, Ampère, Boole, Darboux, Hamburger, \&c., with instructive comparisons, and examples worked out each way; together with a chapter on general transformation, embodying the most important of Bäckland's results. As an example of the power of Lie's methods even in the production of beautiful particular theorems, the proposition on p. 295 may be quoted :-

"When an equation of the second order (of the Monge-Ampere form) has two independent intermediate integrals, it is reducible to the form $s=0$ by contact transformations.'

Very little, comparatively, has been done for equations of order higher than the second. Prof. Lloyd Tanner is one of the few pioneers in this region, and his results, obtained by a different method, are explained in chapter xxii.

Prof. Forsyth explains in his preface and final remarks the principles which have guided him in his choice of material. This must, indeed, have been a most difficult task. It would be easy to dub this treatise " encyclopædic," but it is not, and the fact that it is not is one of its merits. The literature on ordinary linear equations alone which has been published since Fuchs's memoir appeared in Crelle's Journal would much more than fill the whole of Prof. Forsyth's pages. No one who is not prepared to devote the whole of his time to the subject can possibly becom familiar with all that has been written about it; and even if, as is quite possible, this treatise may occasionally disappoint those who consult it on some subsection of the subject in which they are specially interested, it is sure to be of great service by presenting an ordered and not unwieldy body of doctrine, together. with suggestions of the directions in which further progress may be expected. G. B. M

No. 1950 , voL. 75] 